\title{
First arrival traveltime tomography for complex near-surface velocity structures
}

\author{
Mark Noble (Mines ParisTech), Nidhal Belayouni (Mines \\ Paris Tech) and Hervé Chauris (Mines ParisTech)
}

\section{Summary}

Classical algorithms used for travel time tomography are not necessarily well-suited for handling very large seismic data sets, densely parameterized velocity models and for taking advantage of current supercomputers architecture. We revisit the classical approach of firstarrival travel time tomography by proposing to use a simple gradient-based approach without the need of estimating the inverse of the Hessian of the misfit function. The gradient-based approach is very attractive from a numerical point as it can handle large data sets and velocity models and is very robust with respect to the choice of the initial velocity model. 
The tomographic determination of the near-surface velocity structure is a key step when trying to image deeper structures. In most cases, refraction tomography or more generally first-arrival travel time tomography based on direct, diffracted, refracted or diving waves is used to assess a velocity model of the subsurface that best explains the data. For now many years, such algorithms have proved to be valuable tools for obtaining the shallow structure information needed for seismic reflection data processing and imaging.

Current seismic acquisition surveys now commonly deploy thousands of sources combined with thousands of receivers leading to millions or even more acquired traces. Moreover the area under investigation could be very large and this could lead to a velocity model containing millions of parameters whatever the type of parameterization. In addition, the properties of the near-surface that we are trying to image, can exhibit strong vertical and horizontal velocity variations and steep topography, both requiring a velocity model with very fine grids. For 2D geometries, classical first-arrival tomography algorithms may not suffer from computational limitations. On the contrary, for 3D geometries, the Fréchet derivative matrix turns out to be very difficult to handle in terms of memory requirements, even if sparsity is accounted for. To overcome this limitation, one could reduce the size of the data to be inverted or the number of parameters of the model, both leading to either a loss of information or to a poorer resolution.

We can address these issues with the use of a steepest descent method to compute the gradient of the misfit function. For large data sets and densely parameterized velocity models, a gradient based algorithm is very attractive from a numerical point of view as its associated cost is equivalent to the resolution of the forward modeling problem, whatever the size of the input data and the number of unknown velocity parameters. Even if a steepest descent gradient method does require a few more iterations to converge to a final model, this overhead is largely compensated by the fact that parallelization on a supercomputer is straight forward.

In this work, we briefly present our gradient-based algorithm, and show a practical implementation on a complex 2D synthetic model, that exhibits a sharp topography and very strong velocity variations horizontally and vertically.
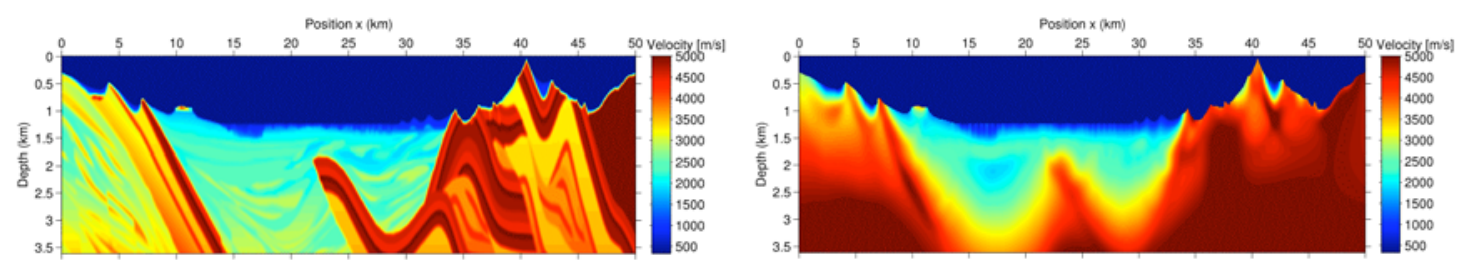

Figure 1: 2D example: True (left), inverted (right) velocity models.
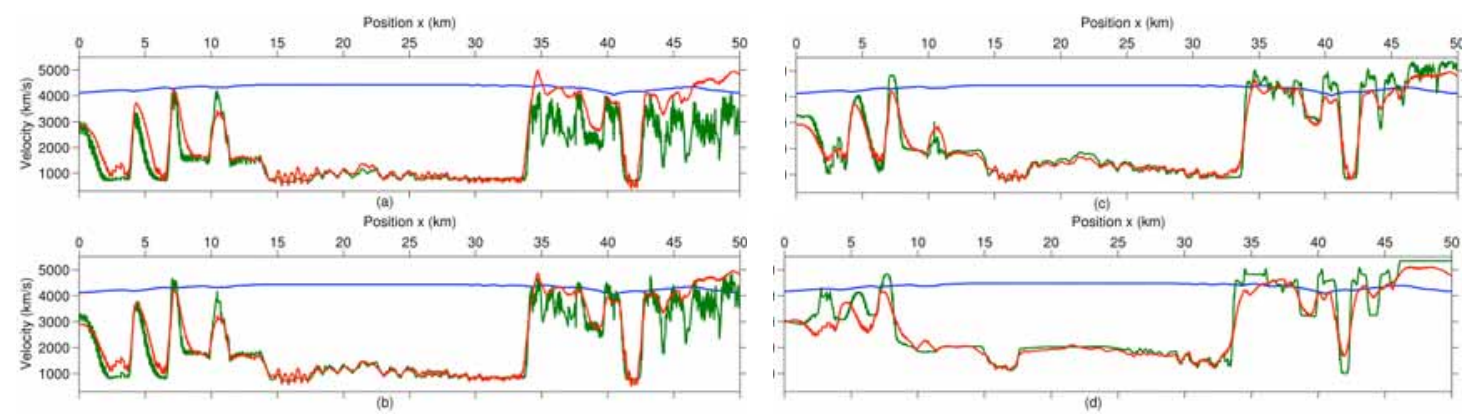

Figure 2: Velocity profiles for the initial (blue), inverted (red) and true (green) velocity models at constant depths bellow the topography, respectively 40 (a), 60 (b), 100 (c), 200 (d).

Acknowledgments: The synthetic velocity model used in the application section was created by Mike O'Brien and Carl Regone and is provided courtesy of Amoco and BP, (http://software.seg.org). 